\title{
ERDÖS-GALLAI-TYPE RESULTS FOR TOTAL MONOCHROMATIC CONNECTION OF GRAPHS
}

\author{
Hui JiAng ${ }^{1}$, XUeliang Li ${ }^{1,2}$ \\ AND \\ YINGYING ZHANG ${ }^{1}$ \\ ${ }^{1}$ Center for Combinatorics and LPMC \\ Nankai University \\ Tianjin 300071, China \\ ${ }^{2}$ School of Mathematics and Statistics \\ Qinghai Normal University \\ Xining, Qinghai 810008, China \\ e-mail: jhuink@163.com \\ lxl@nankai.edu.cn \\ zyydlwyx@163.com
}

\begin{abstract}
A graph is said to be total-colored if all the edges and the vertices of the graph are colored. A total-coloring of a graph is a total monochromaticallyconnecting coloring (TMC-coloring, for short) if any two vertices of the graph are connected by a path whose edges and internal vertices have the same color. For a connected graph $G$, the total monochromatic connection number, denoted by $\operatorname{tmc}(G)$, is defined as the maximum number of colors used in a TMC-coloring of $G$. In this paper, we study two kinds of Erdős-Gallai-type problems for $\operatorname{tmc}(G)$ and completely solve them.
\end{abstract}

Keywords: total-colored graph, total monochromatic connection, ErdősGallai-type problem.

2010 Mathematics Subject Classification: 05C15, 05C35, 05C38, 05C40.

\section{REFERENCES}

[1] J.A. Bondy, U.S.R. Murty, Graph Theory (Springer, 2008).

[2] Q. Cai, X. Li and D. Wu, Erdös-Gallai-type results for colorful monochromatic connectivity of a graph, J. Comb. Optim. 33 (2017) 123-131. doi:10.1007/s10878-015-9938-y 
[3] Q. Cai, X. Li and D. Wu, Some extremal results on the colorful monochromatic vertex-connectivity of a graph. arXiv: $1503.08941 \mathrm{v} 1$.

[4] Y. Caro and R. Yuster, Colorful monochromatic connectivity, Discrete Math. 311 (2011) $1786-1792$. doi:10.1016/j.disc.2011.04.020

[5] G. Ding, T. Hohnson and P. Seymour, Spanning trees with many leaves, J. Graph Theory 37 (2001) 189-197. doi:10.1002/jgt.1013

[6] D. González-Moreno, M. Guevara and J.J. Montellano-Ballesteros, Monochromatic connecting colorings in strongly connected oriented graphs, Discrete Math. 340 (2017) $578-584$. doi:10.1016/j.disc.2016.11.016

[7] R. Gu, X. Li, Z. Qin and Y. Zhao, More on the colorful monochromatic connectivity, Bull. Malays. Math. Sci. Soc. 40 (2017) 1769-1779. doi:10.1007/s40840-015-0274-2

[8] H. Jiang, X. Li and Y. Zhang, More on total monochromatic connection of graphs, Ars Combin. 136 (2018), to appear.

[9] H. Jiang, X. Li and Y. Zhang, Total monochromatic connection of graphs, Discrete Math. 340 (2017) 175-180. doi:10.1016/j.disc.2016.08.020

[10] H. Jiang, X. Li and Y. Zhang, Upper bounds for the total rainbow connection of graphs, J. Comb. Optim. 32 (2016) 260-266. doi:10.1007/s10878-015-9874-x

[11] H. Liu, Â. Mestre and T. Sousa, Total rainbow $k$-connection in graphs, Discrete Appl. Math. 174 (2014) 92-101. doi:10.1016/j.dam.2014.04.012

[12] Y. Sun, On rainbow total-coloring of a graph, Discrete Appl. Math. 194 (2015) $171-177$. doi:10.1016/j.dam.2015.05.012

Received 29 March 2017

Revised 14 November 2017 Accepted 20 November 2017 